Check for updates

Cite this: J. Mater. Chem. C, 2022, 10, 463

Received 18th October 2021, Accepted 8th December 2021

DOI: $10.1039 / \mathrm{d} 1 \mathrm{tc} 05023 \mathrm{k}$

rsc.li/materials-c

\section{Modular chiral Eu(III) complexes for efficient circularly polarized OLEDs $\dagger$}

\author{
Francesco Zinna, (D) *a Lorenzo Arrico, (D) ${ }^{a}$ Tiziana Funaioli, ${ }^{a}$ Lorenzo Di Bari, (D) *a \\ Mariacecilia Pasini, (iD ${ }^{b}$ Chiara Botta ${ }^{b}$ and Umberto Giovanella (DD *b
}

\begin{abstract}
Achieving both high dissymmetry factors and strong emission in circularly polarized (CP) luminescent materials and, at the same time, compatibility with manufacturing processes for organic electronic devices, is a crucial issue for reliable applications of CP emitters in many fields, such as chiral electronics and optoelectronics. In this communication, we show that the independent choice of the sensitizing and the chirality inducing ligands allows europium(III) complexes to meet the multiple requirements for solution processed efficient CP electroluminescent devices.
\end{abstract}

Materials emitting circularly polarized (CP) luminescence ${ }^{1-5}$ are attracting multidisciplinary interest, as they open the way to various applications in the field of enantiospecific (bio)assays, telecommunication, spin-selective devices, etc. ${ }^{6,7}$

In the context of chiral electronics and optoelectronics, in the last few years, several research groups have explored the possibility of obtaining $\mathrm{CP}$ electroluminescence (EL) from Organic Light-Emitting Diodes (OLED) by employing chiral (non-racemic) emitters in the active layer. ${ }^{2,8}$ Such devices are promising for applications in 3D panels, more efficient anti-glare displays, as well as in optical data storage and encryption. ${ }^{9}$

Several successful examples of CP-OLEDs have been reported in recent years, in which CP-EL is obtained through different approaches, ${ }^{1,9}$ i.e. by using single organic molecules, ${ }^{10}$ $\mathrm{d}^{11}$ or $\mathrm{f}^{12,13}$ metal complexes, or supramolecular systems. ${ }^{14}$ On the other hand, several challenges, often difficult to meet all at the same time, remain open: (i) maximizing EL efficiency, (ii) maximizing the circular polarization output (quantified by the dissymmetry factor $\mathrm{g}$ ), (iii) low-cost device manufacturing with extended stability, (iv) availability of the chiral emitters at the required scale.

\footnotetext{
${ }^{a}$ Dipartimento di Chimica e Chimica Industriale, Università di Pisa, via Moruzzi 13, 56124, Pisa, Italy. E-mail: francesco.zinna@unipi.it, lorenzo.dibari@unipi.it

${ }^{b}$ CNR-SCITEC via A. Corti 12, 20133, Milano, Italy.

E-mail: umberto.giovanella@scitec.cnr.it

$\dagger$ Electronic supplementary information (ESI) available. See DOI: 10.1039/ d1 tc05023k
}

Lately, our groups have investigated the use of chiral lanthanide complexes (in particular, Europium) as intrinsic chiral emitters in the active layer of OLEDs, obtaining remarkable results in terms of EL dissymmetry factor $\left(g_{\mathrm{el}}=2\left(I_{\mathrm{L}}-I_{\mathrm{R}}\right) /\left(I_{\mathrm{L}}+I_{\mathrm{R}}\right), I_{\mathrm{L}}\right.$ and $I_{\mathrm{R}}$ being the left and right CP component of EL). ${ }^{12,13}$ Indeed, using chiral lanthanide emitters offers several advantages, such as high dissymmetry factors, high photoluminescence (PL) quantum yield (PLQY) with narrow bands, possibility of deriving effective ligands from the natural chiral pool and facile compound preparation. ${ }^{15}$ Another feature offered by lanthanide systems are bands with opposite polarization, which may be desirable in certain applications, such as near-field multiplexed communications. On the other hand, extracting high EL efficiency from lanthanide-based CP-OLEDs still remains a challenge. A possible solution to these issues can be found in the coordination chemistry of lanthanides. In fact, the high coordination number of lanthanides and the possibility of preparing heteroleptic complexes ${ }^{16}$ allow one to tune the desired properties by using a modular approach.

Indeed, different ligands, each performing a different role, can be chosen thus tuning the required features (partially) independently one from the other. For our purposes, the ligands must fulfil two essential functions: an efficient luminescence sensitization (antenna effect) ensuring, as well, a good radiative exciton recombination, and an effective chiral induction. In this work, we manufacture and investigate CP-OLEDs devices embedding three different Eu(III) complexes (hereafter called Eu1-3, Scheme 1) obtained by choosing among two achiral diketonate ligands, functioning as sensitizers (or antennae), combined with one of two chiral ligands to modulate the complex stereochemistry and therefore its CP emission.

Eu1 is formed by hexafluoroacetylacetonate (HFA) and 2,6bis(4-isopropyl-2-oxazolin-2-yl)pyridine (iPrPyBox), ${ }^{17}$ while Eu2 and Eu3 feature thenoyltrifluoroacetone (TTA) and iPrPyBox and 2,6-bis(4-phenyl-2-oxazolin-2-yl)pyridine (PhPyBox) respectively (Scheme 1). ${ }^{17,18}$ Both HFA and TTA diketonates are efficient sensitizers for $\mathrm{Eu}^{19,20}$ and the modified tridentate pyridine bisoxazoline (PyBox) ligands have proven effective in inducing 

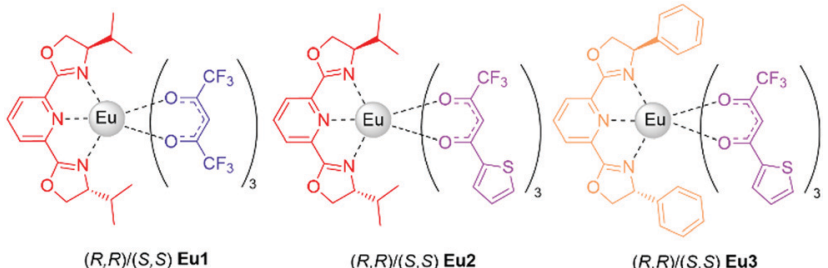

Scheme 1 Chemical structure of chiral Eu(III) complexes used in this work.

highly CP-PL in this type of complexes in solution. A full characterization of the compounds is reported elsewhere. ${ }^{17,18}$

The optical properties of the three Eu(III) complexes (Fig. 1 and Table 1) are studied in toluene solutions and solid-state (thin film) samples by absorption, steady-state PL and timeresolved (TrPL) emission spectroscopy, PLQY, together with their CP-PL activity expressed as the PL dissymmetry factor $g_{\mathrm{pl}}$. Here only the properties of the $S, S$-enantiomer of the three complexes are discussed, while those of the corresponding $R, R$ enantiomer are reported in the ESI. $\dagger$ Then, the complexes are incorporated as intrinsic emitters in the active layer of solution processed OLED architectures.

$\mathrm{Eu}(\mathrm{III})$ complexes in solution exhibit a main absorption band, associated to the $\pi-\pi^{*}$ transition of the $\beta$-diketonate ligand, with a maximum at about $309 \mathrm{~nm}$ for Eu1 and $346 \mathrm{~nm}$ for Eu2 and Eu3 (Fig. S1a-c, ESI $\dagger$ ). The absorption of PyBox ligands falls in the spectral region below $300 \mathrm{~nm}$ (not reported $)^{18}$ and does not play a crucial role in the luminescence sensitization. The optical energy gaps $\left(E_{\mathbf{g}}^{\text {opt }}\right)$ are derived from the onset of absorption spectra. All the $\mathrm{Eu}(\mathrm{III})$ complexes display good PLQYs of about 0.3 , and relatively intense CP-PL activity which shows typical features of $\mathrm{Eu}(\mathrm{III})$ complexes (Fig. S1d-f, ESI $\dagger$ ). We focus the analysis to the $580-630 \mathrm{~nm}$ range where the polarization of the transitions is more relevant. In fact, the strongest circular polarization occurs for the ${ }^{5} \mathrm{D}_{0} \rightarrow{ }^{7} \mathrm{~F}_{1}$ magnetic transition in the range $585-595 \mathrm{~nm}$, while the $g_{\mathrm{pl}}$ is weaker for the ${ }^{5} \mathrm{D}_{0} \rightarrow{ }^{7} \mathrm{~F}_{2}$ electronic transition in the range 610$620 \mathrm{~nm}$ with opposite sign with respect to the magnetic band. It is important to notice that $\mathrm{CP}$ brightness, and therefore the differential output of CP photons, is similar for the two bands in these cases, as often found for Eu complexes. ${ }^{21}$ Indeed, even for the weak ${ }^{5} \mathrm{D}_{0} \rightarrow{ }^{7} \mathrm{~F}_{1}$ transition, such complexes display very large CP brightness factors ${ }^{21}$ (256, 243, $98 \mathrm{M}^{-1} \mathrm{~cm}^{-1}$ for Eu1-3 respectively in toluene solution). Although the $\mathrm{CP}$ brightness as defined in ref. 21 does not directly apply in EL, where no light absorption is involved, the relative intensity of the various ${ }^{5} \mathrm{D}_{0} \rightarrow{ }^{7} \mathrm{~F}_{\mathrm{X}}$ transitions is not significantly different in PL and $\mathrm{EL}$ and moreover the $g_{\mathrm{pl}}$ and $g_{\mathrm{el}}$ factors for the magnetic band are comparable (vide infra). This ensures that the differential flow of photons with opposite circular polarization emitted within the ${ }^{5} \mathrm{D}_{0} \rightarrow{ }^{7} \mathrm{~F}_{1}$ transition remains very relevant even in CP-EL.

$(S, S)$ Eu1 and $(S, S)$ Eu2 show the largest $g_{\mathrm{p} 1}$ values of 0.77 and 0.75 at $592 \mathrm{~nm}$, respectively, while $(S, S)$ Eu3 features $g_{\mathrm{PL}}$ peaks of -0.22 at 592 and $596 \mathrm{~nm}$. Notably, an opposite sense of chirality can be obtained for the same absolute configuration of PyBox ligand bearing different side arms in Eu(III) TTA-based complexes. ${ }^{17,18,22}$ In fact, the sign of the CP-PL for $(S, S)$ Eu1 and Eu2 (both bearing $(S, S)-{ }^{i}$ PrPyBox) is positive for the ${ }^{5} \mathrm{D}_{0} \rightarrow$ ${ }^{7} \mathrm{~F}_{1}$ transition, and it is reversed for $(S, S)$ Eu3. This confirms that the substituents on the chiral ancillary ligand affect the CP emission signature independently from the diketonate used. The photophysics of $\mathrm{Eu}(\mathrm{III})$ complexes dispersed in host matrices are evaluated as thin films in view of their use in the active layer of CP-OLEDs. Firstly, the dispersion of Eu1-3 in a poly(methyl methacrylate) (PMMA) matrix offers the opportunity to study Eu(III) complexes behaviour in rigid and neutral environment.
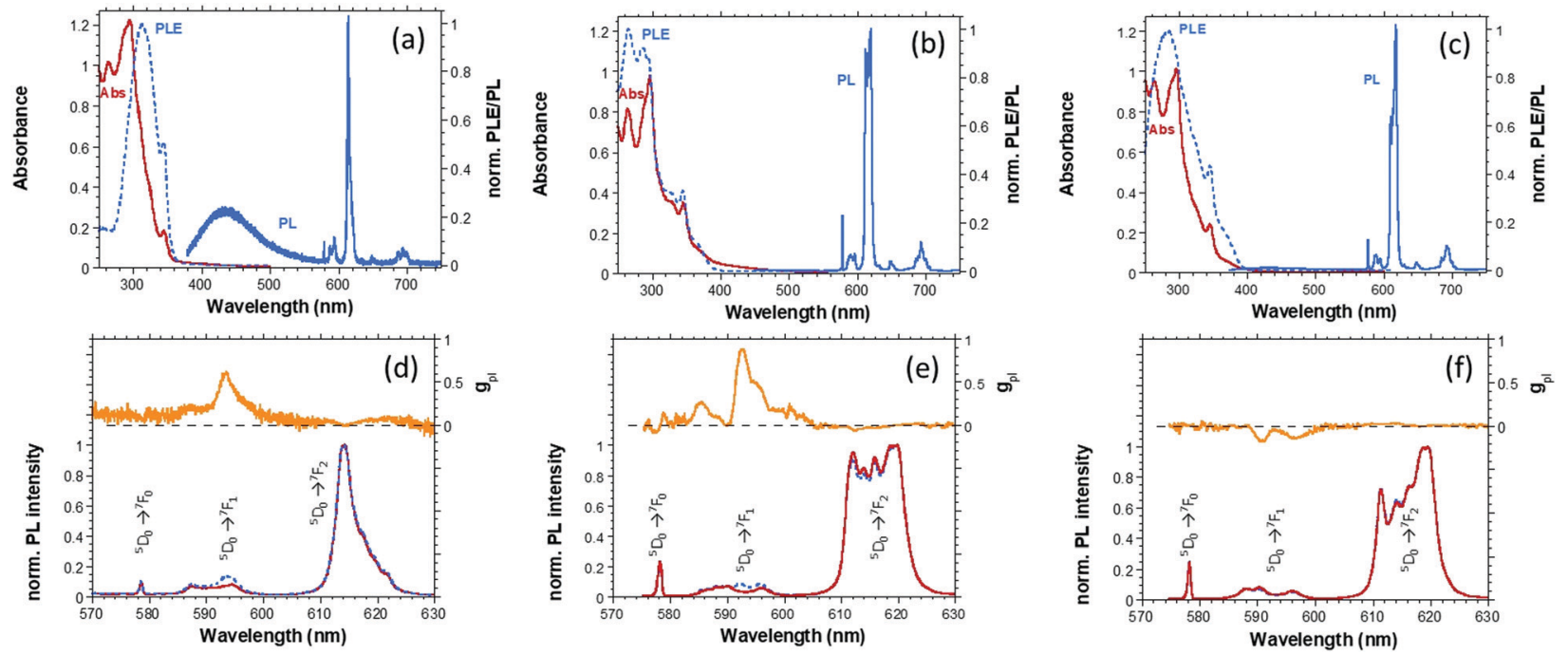

Fig. 1 Photophysical data of PVK:OXD7:Eu(III) complexes films: (a-c) Absorption (solid red line), normalized PLE (dotted blue line), normalized PL (solid blue line), (d-f) CP-PL for the two polarizations (solid red and dashed blue lines) and $g_{p l}$ (orange line) of films embedding $(S, S)$ Eu1 (a,d), $(S, S)$ Eu2 $(b, e)$ and $(S, S) \operatorname{Eu3}(c, f) . \lambda_{\mathrm{ex}}=350 \mathrm{~nm} ; \lambda_{\mathrm{em}}=614 \mathrm{~nm}(\mathrm{a}), \lambda_{\mathrm{em}}=619 \mathrm{~nm}(\mathrm{~b}, \mathrm{c})$. 
Table 1 Summary of optical properties of complexes in toluene solutions, solid-state (thin film) and devices. PL quantum yield (PLQY) measurements, decay times $(\tau)$, together with corresponding dissymmetry factor main peaks. Data reported for $(S, S)$ complexes

\begin{tabular}{|c|c|c|c|c|c|c|c|c|c|}
\hline \multirow[b]{2}{*}{ Compound } & \multicolumn{3}{|c|}{ Solution } & \multicolumn{3}{|l|}{$\frac{\text { Film }}{\text { PMMA }}$} & \multicolumn{2}{|c|}{$\overline{\text { PVK:OXD7 }}$} & \multirow{2}{*}{$\begin{array}{l}\text { Devices }^{d} \\
g_{\text {el }}(\text { peak, nm) }\end{array}$} \\
\hline & $\overline{\mathrm{PLQY}^{a}}$ & $\overline{\tau(\mu \mathrm{s})^{b}}$ & $g_{\mathrm{pl}}($ peak, nm) & $\overline{\mathrm{PLQY}^{c}}$ & $\tau(\mu \mathrm{s})^{b}$ & $g_{\mathrm{pl}}($ peak, nm) & $\tau,(\mu \mathrm{s})^{b}$ & $g_{\mathrm{pl}}($ Peak, nm) & \\
\hline Eu1 & 0.30 & 868 & $\begin{array}{l}0.30(585) \\
0.76(592) \\
-0.02(614)\end{array}$ & 0.52 & 1029 & $\begin{array}{l}0.30(585) \\
0.70(593) \\
-0.02(614)\end{array}$ & 658 & $\begin{array}{l}0.2(585) \\
0.6(593) \\
-\end{array}$ & - \\
\hline Eu2 & 0.30 & 574 & $\begin{array}{l}0.25(585) \\
0.75(592) \\
-0.06(612)\end{array}$ & 0.55 & 689 & $\begin{array}{l}0.21(585) \\
0.59(592) \\
-0.03(612)\end{array}$ & 565 & $\begin{array}{l}0.26(585) \\
0.88(592) \\
-0.06(612)\end{array}$ & $\begin{array}{l}0.16(585) \\
0.51(592) \\
-0.04(612)\end{array}$ \\
\hline Eu3 & 0.32 & 508 & $\begin{array}{l}-0.22(592) \\
-0.22(596) \\
0.04(614)\end{array}$ & 0.62 & 667 & $\begin{array}{l}-0.18(591) \\
-0.17(597) \\
0.03(614)\end{array}$ & 467 & $\begin{array}{l}-0.17(591) \\
-0.15(596) \\
0.03(614)\end{array}$ & $\begin{array}{l}-0.22(591) \\
-0.25(597)\end{array}$ \\
\hline
\end{tabular}

${ }^{a} \lambda_{\mathrm{ex}}=365 \mathrm{~nm} .{ }^{b} \lambda_{\mathrm{ex}}=320 \mathrm{~nm}, \lambda_{\mathrm{em}}=614 \mathrm{~nm}(\mathbf{E u 1}) ; \lambda_{\mathrm{ex}}=350 \mathrm{~nm}, \lambda_{\mathrm{em}}=619 \mathrm{~nm}\left(\right.$ Eu2 , Eu3). ${ }^{c}$ Integrating sphere. ${ }^{d}$ With transparent cathode.

Then the dispersion of Eu1-3 in the active matrix used for OLEDs is investigated. Poly(9-vinylcarbazole) (PVK) together with 1,3-bis[2-(4-tert-butylphenyl)-1,3,4-oxadiazo-5-yl]benzene (OXD7) is the most widely used bipolar host already successfully employed with Ln(III) complexes. ${ }^{13}$ The Eu(III) complexes are dispersed in PVK: OXD7 (whose ratio is set to $3: 1 \mathrm{wt} \%$.) with a fixed concentration of $8.5 \%$ by mass with respect to the matrix.

Absorption spectra of the PMMA blends (Fig. S2a-c, ESI $\dagger$ ) resemble the solutions ones, while spectra of PVK:OXD7:Eu1-3 films (Fig. 1a-c) are dominated by the PVK:OXD7 contribution.

Importantly, the CP activity is retained in the blends (Fig. 1). The ECD spectra of PVK:OXD7:Eu1-3 films (Fig. S3-S5, ESI $\dagger$ ), despite a weak signal due to the low concentration of the complexes in the polymer blend, is similar to the spectra obtained in solution. ${ }^{17,18}$ This indicates that a similar geometry of the complexes is preserved in the active-matrix blend. In particular, in the ECD spectrum of $(R, R)$ Eu3 blend, the main Cotton effect (around $360 \mathrm{~nm}$, associated with TTA $\pi \rightarrow \pi^{*}$ transition) is inverted with respect to the main band observed in the case of $(R, R)$ Eu1 and $(R, R)$ Eu2 $(315$ and $340 \mathrm{~nm}$ respectively). On the other hand, the spectra of $(R, R) \mathbf{E u 1}$ and $(R, R) \mathbf{E u} 2$ blends are comparable, except for the blue shift of Eu1, due to a higher HOMO-LUMO gap in the HFA $v s$. TTA. These data point towards a similar arrangement of the ligands around the metal centre for $\mathbf{E u} 1$ and $\mathbf{E u} 2$ complexes due to the presence of ${ }^{\mathrm{i}}$ PrPyBox, while an almost mirror image coordination geometry of the TTA ligands is at play where the chirality induction is led by PhPyBox, as in the case of Eu3. Moreover, no induced ECD onto the polymer blend is observed, excluding any significant chirality transfer from the complex to the matrix, as it is instead the case with, e.g., some helicene/achiral polymer systems. ${ }^{23}$

The values of $g_{\mathrm{pl}}$ recorded for Eu1 complex dispersed in the blends are slightly lower than in the corresponding toluene solutions (Table 1). $(S, S)$ Eu1 features similar $g_{\mathrm{pl}}$ of 0.7 and 0.6 at $593 \mathrm{~nm}$ in PMMA and PVK:OXD7 blends, respectively, while $(S, S)$ Eu2 shows the highest value once dispersed in PVK:OXD7 (0.88 with respect to 0.59 in PMMA matrix). ( $S, S)$ Eu3 displays lower (and again reverse in sign) $g_{\text {pl }}$ with respect to $(S, S)$ Eu1-2 at two different wavelengths $(-0.17$ and -0.15 at 591 and $597 \mathrm{~nm}$ in PVK:OXD7 blend).
PL excitation spectrum (PLE) of PVK:OXD7:Eu1 film (Fig. 1a), recorded by monitoring the emission at $614 \mathrm{~nm}$, is very similar to the absorption of Eu1 solution (Fig. S1a, ESI $\dagger$ ) mainly associated to HFA transitions, and does not follow the absorption of the film (except for a weak PVK band at $344 \mathrm{~nm}$ ), meaning that negligible Förster resonant energy transfer (FRET) from host (excitation donor) to Eu1 (acceptor) takes place. Accordingly, the broad PVK emission at about $440 \mathrm{~nm}$ is observed in the blend (Fig. 1a) once excited at 310-350 nm. On the contrary, the PLE spectra of both Eu2 and Eu3 blend films (Fig. 1b and c) resemble their absorption spectra (Fig. S1b and c, ESI $\dagger$ ) indicating that efficient FRET occurs from the host matrix, whose emission is completely quenched (Fig. 1b and c). The observed different efficiency of FRET from PVK to the $\mathrm{Eu}$ (III) complexes is consequence of the lack of spectral overlap between Eu1 absorption and PVK emission, while a good overlap is observed for Eu2 or Eu3 (see Fig. S6, ESI $\dagger$ ). Generally, the FRET mechanism promotes the radiative recombination at acceptor sites, hence positively impacting on device performance. However, excitation back transfer from Eu(III) complex ligand to the host triplet energy level represents a major quenching mechanism in PVL:OXD7:Eu(III) complexes films. ${ }^{24}$ This is more critical for Eu1, whose triplet energy $(2.65 \mathrm{eV})$ is higher than the PVK one (2.5 eV, Fig. S8, ESI $\dagger$ ).

TrPLs of the Eu1-3, both in solution and blends, were performed (Fig. S7, ESI $\dagger$ ) to support this scenario. All the complexes display longer lifetimes in the rigid PMMA matrix with respect to the solution, with Eu1 displaying the longest lifetimes among the three complexes (Table 1). The decays are systematically faster in PVK:OXD7 than in PMMA blends. This is particularly evident for complex Eu1, whose lifetime and PLQY (0.24) in the PVK:OXD7 blend decreases by about a factor 2 when compared to the PMMA blend.

This analysis indicates that the diketonate ligand (TTA vs. HFA) plays a main role in both the exciton recombination process and in ET from the polymer host. In particular, among the three complexes, the use of Eu1 in solution processed OLEDs with PVK-based active layers appears unfavourable both for the emission quenching induced by back-transfer to PVK and for the absence of FRET processes. 


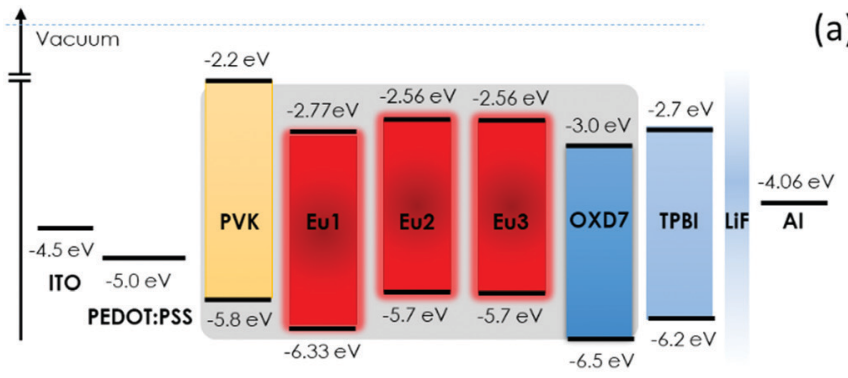

(a)
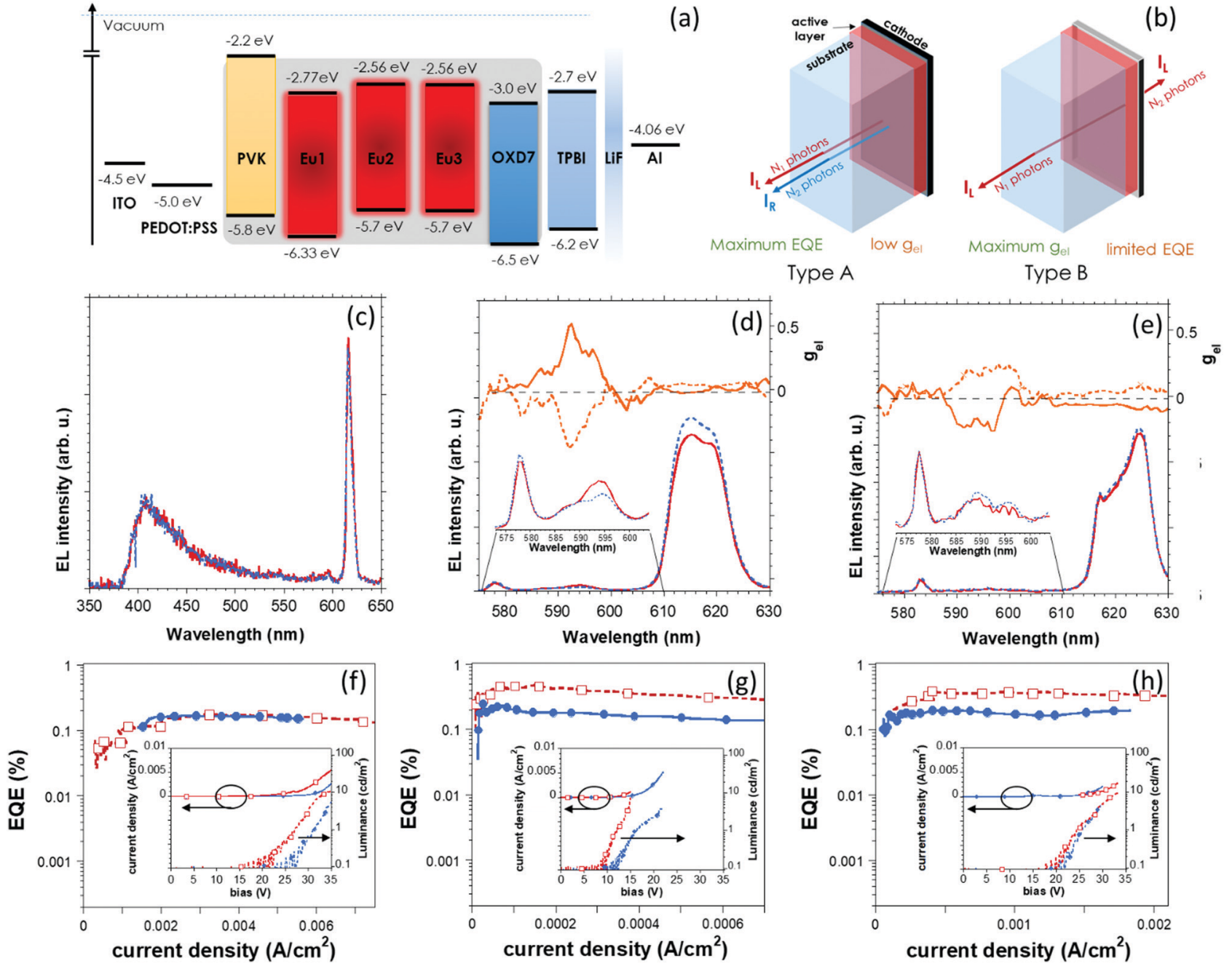

Fig. 2 (a) Flat-band energy level diagram of the CP-OLEDs, (b) Structure of type A (reflective cathode) and type B (transparent cathode) devices; CP-EL spectra (solid red and dashed blue lines for the two CP components) of devices embedding Eu1 (c), Eu2 (d) and Eu3 (e) with transparent cathode (inset: expansion of the EL between 575 and $610 \mathrm{~nm}$ ), and $g_{\mathrm{el}}$ (orange lines, continuous for $(S, S)$, dashed for $(R, R)$ enantiomer) for Eu2-3; corresponding EQE vs. $\mathrm{J}$ of type A (red $\square$ ) and B devices (blue - embedding Eu1 (f), Eu2 (g) and Eu3 (h); in the insets, corresponding current density-voltage curves (solid lines) and Luminance-voltage (dashed lines) of type A (red $\square$ ) and B devices (blue

Cyclic voltammetry of $\mathrm{Eu}(\mathrm{III})$ complexes was performed to obtain molecular energy levels of the complexes from the measurement of their oxidation potentials (Fig. S9, ESI†) and optical gap. The determined LUMO value is -2.77 for Eu1 and $-2.56 \mathrm{eV}$ for Eu2 and Eu3. The HOMO level is -6.33 and $-5.77 \mathrm{eV}$ for Eu1 and Eu2-Eu3 respectively (Fig. 2a).

The devices were then fabricated with the architecture ITO/ PEDOT:PSS/PVK:OXD7:Eu(III) complexes/TPBI/Ba/Al [TPBI: 2,2', $2^{\prime \prime}$-(1,3,5-benzinetriyl)-tris(1-phenyl-1- $H$-benzimidazole)] whose flat band energy level diagram is reported in Fig. 2 a.

The active layer ( $80 \mathrm{~nm}$ thick) is processed by spin-coating from $15 \mathrm{mg} \mathrm{ml}^{-1} \mathrm{CHCl}_{3}$ degassed solution over PEDOT:PSScovered ITO/glass substrate. The formulation of the blend is the same used for the photophysical characterization (the content of Eu(III) complex is fixed to 8.5 wt.\% with respect to the host matrix, see Fig. S7, ESI $\dagger$ ) as it ensures both superior performance of the device and pure Eu-centred EL. A $30 \mathrm{~nm}$ thick TPBI electron transporting/hole blocking layer, thermo- sublimated in high vacuum on top of the active layer, is finally covered by the LiF/Al cathode deposition.

Devices are fabricated with two different strategies to maximize the performance in term of external quantum efficiency (EQE, measured in forward direction) (hereafter called devices of type A) or CP-EL (type B, see Fig. 2b). In fact, since the transparency of the cathode mostly impacts on the EQE of the devices, ${ }^{12,13,25}$ type A devices are designed with a reflective cathode of $1.5 \mathrm{~nm}$ of LiF capped by $110 \mathrm{~nm}$ of aluminium to allow for a maximal collection of the photons in forward direction (maximum EQE). On the other hand, reflection on the back electrode causes a reversal of handedness of CP light and therefore a decrease of polarization performances. Thus, in devices of type $\mathrm{B}$, the thickness of aluminium capping is reduced to $6 \mathrm{~nm}$ (while LiF remained unchanged) to be almost transparent to red light and to provide higher EL dissymmetry factors. All the devices exhibit EL spectra with typical features of Eu(III) emission. As expected, the higher EQE, measured in 
forward direction, is observed in type A devices (Fig. S10, ESI $\dagger$ ). ${ }^{12}$ EQE of Eu2-based devices reaches the value of $0.48 \%$, which is the highest reported for chiral Europium-based OLEDs and exceeds of one order of magnitude the earlier reported results. ${ }^{13}$ In corresponding type B devices, the EQE is slightly lower $(0.22 \%)$, but a remarkable EL dissymmetry factor $\mathrm{g}_{\mathrm{el}}$ of $\sim 0.51$ [for (S,S) enantiomer] indicates that at $592 \mathrm{~nm}$ (Fig. 2d), a high degree of CP photons (63\% of them are left polarized) can be achieved in EL as well, once the reflection on the back electrode is reduced (Table 1). Type A devices based on Eu1 and Eu3 showed EQE of $0.17 \%$ and $0.38 \%$ respectively. These figures are roughly in the range of the ones reported for regular (i.e. non-polarized) OLEDs based on $\mathrm{Eu}$ complexes. ${ }^{26} \mathrm{As}$ expected from CP-PL spectra, with the same absolute configuration of the PyBox ligand, Eu3-based devices show opposite sign $\mathrm{g}_{\mathrm{el}}$ with respect to Eu2-based ones (namely $\mathrm{g}_{\mathrm{el}}+0.51 /-0.25$ for ${ }^{5} \mathrm{D}_{0} \rightarrow{ }^{7} \mathrm{~F}_{1}$ transition in the case of $(S, S)$ Eu2 and Eu3 respectively, Fig. 2d, e and Table 1). CP-EL spectra obtained for devices containing enantiomer complexes are mirror image, ensuring the reliability of the polarization data measured for the devices.

It must be noted that good PLQYs measured for Eu1-3 does not guarantee good emission performance in a device. In fact, the low HOMO level of Eu1 with respect to Eu2 and Eu3 most probably prevents direct charge trapping upon biasing, and, together with the observed lack of FRET, promotes a contribution of the PVK blue emission to the EL spectrum (see Fig. 2c), affecting the performance of the device. Moreover, the high driving voltages of Eu1-based devices (20-25 V, Fig. S11, ESI $\dagger$ ), due to larger hole injection energy barriers of Eu1 with respect to Eu2-3 (Fig. 2a), causes unstable emission that prevents reliable determination of $\mathrm{g}_{\mathrm{el}}$. Further, the significant reduction of PL decay time observed in Eu1,3 blended in PVK:OXD7 matrix, compared to PMMA, suggests that PVK:OXD7 is energetically not enough well-suited ${ }^{27}$ to dilute Eu1,3 with respect to Eu2. All devices show low roll-off efficiency, but a modest luminance (10 $\mathrm{cd} \mathrm{m}^{-2}$ at about $15 \mathrm{~V}$ for Eu2 and 30-35 V for Eu1,3-based devices), leaving room for an improvement by exploring different host matrices. ${ }^{14,26}$

\section{Conclusions}

In conclusion, we obtained CP-OLED devices with peculiar architectures based on Ln(III) complexes showing both remarkable emission efficiency $(\mathrm{EQE}=0.48 \%$ ) and polarization performances $\left(\left|g_{\text {el }}\right|=0.51\right)$. This was achieved by taking advantage of a modular strategy for the design of the Ln(III) emitter. Lanthanide chemistry allows for the easy preparation of heteroleptic complexes, that is of complexes containing two different ligands. In the first place, three diketonates are used to sensitize Ln(III) emission, while providing stable coordination and a neutral core. Although from a spectroscopic point of view TTA and HFA may be considered similar, the ability of the former to harvest exciton recombination events in the OLED architecture developed herein is far superior, compared to the latter. Secondly, a chiral ligand with nitrogen donors completes the Ln(III) coordination sphere, endowing the complex with the necessary dissymmetry and controlling the circular polarization of the device. We believe that such modular strategy can be effective to adapt lanthanide complexes to the multiple requirements of efficient CP-OLEDs.

\section{Author contributions}

FZ and LDB conceived the idea, FZ and LA prepared the complexes and performed preliminary (chiro)optical characterization, UG manufactured CP-OLEDs and carried out device characterization. MP optimized the formulation of the blends and performed film deposition. CB performed the photophysical characterization. TF performed electrochemical analysis. FZ, UG wrote the manuscript, all the authors read and approved the final version.

\section{Conflicts of interest}

There are no conflicts to declare.

\section{Acknowledgements}

Financial support from Italian University and Research Ministry (PRIN Project 20172M3K5N) is gratefully acknowledged. FZ and LDB thank the University of Pisa for financial support (PRA 2020_21 and 2019_23).

\section{References}

1 Y. Deng, M. Wang, Y. Zhuang, S. Liu, W. Huang and Q. Zhao, Light: Sci. Appl., 2021, 10, 76.

2 H. Kuang, C. Xu and Z. Tang, Adv. Mater., 2020, 32, 2005110.

3 G. Yang, M. Kazes and D. Oron, Adv. Funct. Mater., 2018, 28, 1802012.

4 S. Ma, J. Ahn and J. Moon, Adv. Mater., 2021, 2005760.

5 T. Mori, "Circularly Polarized Luminescence of Isolated Small Organic Molecules”, Springer, 2020.

6 J. R. Brandt, F. Salerno and M. J. Fuchter, Nat. Rev. Chem., 2017, 1, 0045.

7 R. Farshchi, M. Ramsteiner, J. Herfort, A. Tahraoui and H. T. Grahn, Appl. Phys. Lett., 2011, 98, 162508.

8 J. M. Han, S. Guo, H. Lu, S. J. Liu, Q. Zhao and W. Huang, Adv. Opt. Mater., 2018, 6, 1800538.

9 D.-W. Zhang, M. Li and C.-F. Chen, Chem. Soc. Rev., 2020, 49, 1331-1343 and reference therein.

10 X. Zhang, Y. Zhang, Y. Li, Y. Quan, Y. Cheng and Y. Li, Chem. Commun., 2019, 55, 9845; Z.-G. Wu, H.-B. Han, Z.-P. Yan, X.-F. Luo, Y. Wang, Y.-X. Zheng, J.-L. Zuo and Y. Pan, Adv. Mater., 2019, 31, 1900524; M. Li, Y.-F. Wang, D. Zhang, L. Duan and C.-F. Chen, Angew. Chem., Int. Ed., 2020, 59, 3500; L. Frédéric, A. Desmarchelier, R. Plais, L. Lavnevich, G. Muller, C. Villafuerte, G. Clavier, E. Quesnel, B. Racine, S. Meunier-Della-Gatta, J.-P. Dognon, P. Thuéry, J. Crassous, L. Favereau and G. Pieters, Adv. Funct. Mater., 2020, 30, 2004838; Y.-P. Zhang, X. Liang, X.-F. Luo, 
S.-Q. Song, S. Li, Y. Wang, Z.-P. Mao, W.-Y. Xu, Y.-X. Zheng, J.-L. Zuo and Y. Pan, Angew. Chem., Int. Ed., 2021, 60, 8435; K. Dhbaibi, L. Abella, S. Meunier-Della-Gatta, T. Roisnel, N. Vanthuyne, B. Jamoussi, G. Pieters, B. Racine, E. Quesnel, J. Autschbach, J. Crassous and L. Favereau, Chem. Sci., 2021, 12, 5522-5533; M. Li, M.-Y. Wang, Y.-F. Wang, L. Feng and C.-F. Chen, Angew. Chem., Int. Ed., 2021, 60, 20728.

11 T. Y. Li, Y. M. Jing, X. Liu, Y. Zhao, Y.-X. Zheng and J.-L. Zuo, Sci. Rep., 2015, 5, 14912; J. M. Han, S. Guo, J. Wang, L. W. Wei, Y. L. Zhuang, S. J. Liu, Q. Zhao, X. W. Zhang and W. Huang, Adv. Opt. Mater., 2017, 5, 1700359; Z.-P. Yan, X.-F. Luo, W.-Q. Liu, Z.-G. Wu, X. Liang, K. Liao, Y. Wang, Y.-X. Zheng, L. Zhou, J.-L. Zuo, Y. Pan and H. Zhang, Chem. - Eur. J., 2019, 25, 5672; Z.-P. Yan, K. Liao, H.-B. Han, J. Su, Y.-X. Zheng and J.-L. Zuo, Chem. Commun., 2019, 55, 8215; Y. Chen, X. Li, N. Li, Y. Quan, Y. Cheng and Y. Tang, Mater. Chem. Front., 2019, 3, 867; G. Lu, Z.-G. Wu, R. Wu, X. Cao, L. Zhou, Y.-X. Zheng and C. Yang, Adv. Funct. Mater., 2021, 31, 2102898.

12 F. Zinna, U. Giovanella and L. Di Bari, Adv. Mater., 2015, 27, 1791.

13 F. Zinna, M. Pasini, F. Galeotti, C. Botta, L. Di Bari and U. Giovanella, Adv. Funct. Mater., 2017, 27, 1603719.

14 Y. Yang, R. C. da Costa, D.-M. Smilgies, A. J. Campbell and M. J. Fuchter, Adv. Mater., 2013, 25, 2624; J. R. Brandt, X. Wang, Y. Yang, A. J. Campbell and M. J. Fuchter, J. Am. Chem. Soc., 2016, 138(31), 9743; D.-M. Lee, J.-W. Song, Y.-J. Lee, C.-J. Yu and J.-H. Kim, Adv. Mater., 2017, 29, 1700907; D. Di Nuzzo, C. Kulkarni, B. Zhao, E. Smolinsky, F. Tassinari, S. C. J. Meskers, R. Naaman, E. W. Meijer and R. H. Friend, ACS Nano, 2017, 11(12), 12713; F. Song, Z. Xu, Q. Zhang, Z. Zhao, H. Zhang,
W. Zhao, Z. Qiu, C. Qi, H. Zhang, H. H. Y. Sung, I. D. Williams, J. W. Y. Lam, Z. Zhao, A. Qin, D. Ma and B. Z. Tang, Adv. Funct. Mater., 2018, 28, 1800051.

15 F. Zinna and L. Di Bari, Chirality, 2015, 27, 1; H.-Y. Wong, W.-S. Lo, K.-H. Yim and G.-L. Law, Chem, 2019, 5(12), 3058; L. E. MacKenzie and R. Pal, Nat. Rev. Chem., 2021, 5, 109.

16 H. Xu, Q. Sun, Z. An, Y. Wei and X. Liu, Coord. Chem. Rev., 2015, 293-294, 228.

17 J. Yuasa, T. Ohno, K. Miyata, H. Tsumatori, Y. Hasegawa and T. Kawai, J. Am. Chem. Soc., 2011, 133, 9892.

18 M. Górecki, L. Carpita, L. Arrico, F. Zinna and L. Di Bari, Dalton Trans., 2018, 47, 7166.

19 J.-C. G. Bünzli and S. V. Eliseeva, in Basics of Lanthanide Photophysics. Lanthanide Luminescence: Photophysical, Analytical and Biological Aspects, ed. P. Hänninen and H. Härmä, Springer, Heidelberg, 2010, p. 1.

20 C. Freund, W. Porzio, U. Giovanella, F. Vignali, M. Pasini, S. Destri, A. Mech, S. Di Pietro, L. Di Bari and P. Mineo, Inorg. Chem., 2011, 50, 5417.

21 L. Arrico, L. Di Bari and F. Zinna, Chem. - Eur. J., 2021, 27, 2920; Y. Nagata and T. Mori, Front. Chem., 2020, 8, 448.

22 F. Zinna, L. Arrico and L. Di Bari, Chem. Commun., 2019, 55, 6607.

23 J. Wade, J. R. Brandt, D. Reger, F. Zinna, K. Y. Amsharov, N. Jux, D. L. Andrews and M. J. Fuchter, Angew. Chem., Int. Ed., 2021, 60, 222.

24 U. Giovanella, M. Pasini, C. Freund, C. Botta, W. Porzio and S. Destri, J. Phys. Chem. C, 2009, 113(6), 2290.

25 F. Zinna, G. Pescitelli and L. Di Bari, Chirality, 2020, 32, 765.

26 L. Wang, Z. Zhao, C. Wei, H. Wei, Z. Liu, Z. Bian and C. Huang, Adv. Opt. Mater., 2019, 7, 1801256.

27 T. W. Canzler and J. Kido, Org. Electron., 2006, 7, 29. 